\title{
PRODUÇÃO CIENTIFICA EM PEDIATRIA NO PERÍODO DE 2000 A 2007
}

\author{
Ana Maria Dyniewicz ${ }^{1}$, Janislei Giseli Dorociaki Stocco², Nadine de Biagi Ziesemer ${ }^{3}$
}

\begin{abstract}
RESUMO: A investigação amplia conhecimentos, promove mudanças na prática, estimula maior reflexão e crítica, caracterizando a Enfermagem como disciplina do saber. O objetivo foi analisar a produção científica em enfermagem pediátrica, por meio de pesquisa descritiva, em resumos publicados em periódicos, teses e dissertações no período de janeiro de 2000 a outubro de 2007. Foram selecionados 237 resumos, 179 artigos em periódicos, 46 dissertações e 09 teses. Os dados foram categorizados por título do periódico; ano e tipo de publicação e áreas temáticas: assistencial; administrativa; relatos de experiência; ensino e pesquisa; profissional e reflexões teóricas. Os resultados mostraram produções predominantemente na área assistencial, com temas relacionados à criança, às mães e à equipe de enfermagem, com incremento de publicações a partir de 2003. Conclui-se que esta ocorrendo ampliação da investigação pediátrica, pois é pelo conhecimento sistematizado e experiências profissionais que se revelam atributos de competência e de responsabilidade social.
\end{abstract}

PALAVRAS CHAVE: Enfermagem; Pediatria; Publicações periódicas.

\section{PEDIATRIC SCIENTIFIC PRODUCTION IN THE PERIOD 2000 TO 2007}

\begin{abstract}
Investigation expands knowledge, promotes changes in practice, stimulates further reflection and critique, characterizing Nursing as a discipline of knowledge. The objective was to assess scientific production in pediatric nursing, by means of descriptive research, in abstracts published in the period from January 2000 through October 2007. The selection comprised 237 abstracts, 179 journal articles, 46 dissertations and 09 theses. The data were categorized by journal title; year and type of publication, and the following thematic areas: nursing care; administrative; experience reports; teaching and research; professional, and theoretical reflections. The results showed predominance of productions in the area of nursing care, with topics related to children, mothers and the nursing team, and an increase in the number of publications as from 2003. It was concluded that an expansion of investigation is taking place in pediatrics, for it is through systematic knowledge and professional experiences that attributes of competence and social responsibility are revealed. KEYWORDS: Nursing; Pediatrics; Periodicals.
\end{abstract}

\section{PRODUCCIÓN CIENTÍFICA EN PEDIATRIA EN EL PERÍODO DE 2000 A 2007}

RESUMEN: La investigación amplía conocimientos, promueve cambios en la práctica, fomenta una mayor reflexión y crítica, caracterizando la Enfermería como disciplina del saber. El objetivo fue analizar la producción científica en enfermería pediátrica, a través de la investigación descriptiva, en resúmenes publicados en periódicos, tesis y disertaciones en el periodo de enero de 2000 a octubre de 2007. Se seleccionaron 237 resúmenes, 179 artículos en periódicos, 46 disertaciones y 09 tesis. Los datos fueron analizados por categoría de título del periódico, año y tipo de publicación y áreas temáticas: asistencial, administrativa, relatos de experiencia, enseñanza e investigación; profesional y reflexiones teóricas. Los resultados mostraron producciones predominantemente en el área asistencial, con temas relacionados a los niños, a las madres y al equipo de enfermería, con un aumento de publicaciones a partir de 2003. De ello se deduce que está ocurriendo expansión de la investigación en pediátrica, pues es por el conocimiento sistemático y las experiencias profesionales que se revelan atributos de competencia y de responsabilidad social.

PALABRAS CLAVE: Enfermería; Pediatría; Publicaciones periódicas.

${ }^{1}$ Enfermeira. Doutora em Enfermagem-UNIFESP. Docente de pós graduação Universidade Tuiuti do Paraná.

${ }^{2}$ Enfermeira. Mestranda em Enfermagem pelo Programa de Pós-Graduação em Enfermagem da Universidade Federal do Paraná-UFPR. Enfermeira da Unidade de Terapia Intensiva Pediátrica do Hospital de Clínicas-UFPR.

${ }^{3}$ Enfermeira. Mestre em Enfermagem-UNIFESP com ênfase em Enfermagem Pediátrica. Docente da Faculdade Evangélica do ParanáFEPAR.

Autor correspondente:

Ana Maria Dyniewicz

Rua Pe Anchieta, 1965 - 80730-000 - Curitiba-PR

Recebido: 18/07/08

E.mail: anadyni@yahoo.com.br

Aprovado: 26/02/09

Cogitare Enferm 2009 Jan/Mar; 14(1):144-9 


\section{INTRODUÇÃO}

A investigação em Enfermagem aproxima o saber construído por enfermeiros do conhecimento científico. É caminho para rever práticas, estabelecer estratégias e proceder mudanças teórico-metodológicas e, seguramente, é mais uma forma de manter, aumentar ou melhorar competências, compatíveis com as exigências da responsabilidade social da profissão.

No Brasil a produção científica torna-se mais evidente com a criação dos cursos de pós-graduação, a partir da década de 70 , conforme o previsto na lei 5.540/68 da Reforma Universitária ${ }^{(1)}$. Nesse cenário, ocorre a criação de cursos de Pós-Graduação na área da Enfermagem Pediátrica, destacando-se o curso da Escola Paulista de Enfermagem, atual Departamento de Enfermagem da Universidade Federal de São Paulo(2).

Outro fator importante é o paulatino comprometimento de enfermeiros da prática e docentes com relação à melhoria da qualidade da prática de enfermagem por meio da pesquisa, ou seja, a teorização requerendo sistematização para enriquecêla. Afinal, no desenvolvimento de pesquisa utiliza-se a razão para organizar a realidade tornando-a compreensível. Dessa forma, a realidade, embasada por princípios teóricos e filosóficos, indica o que se necessita estudar e conhecer, a partir disso delimitase o que se deseja investigar ${ }^{(3)}$.

Especificamente, em enfermagem pediátrica o cenário hospitalar é desconhecido pela criança que, uma vez internada, passa a conviver com outras crianças em diferentes estágios de enfermidade, sendo transportada para um ambiente associado ao sofrimento e, algumas vezes, à morte. É nesse ambiente que enfermeiro e a criança trocam suas experiências, sua cultura, seus valores, seu subjetivismo, seus interesses e suas expectativas ${ }^{(4)}$, as quais podem e devem ser transformadas em estudos que possam ser socializados com a comunidade científica.

A socialização do saber em enfermagem pediátrica em seu saber técnico, perpassa o processo de humanização do cuidado, da dignidade humana, do respeito e valorização da vida e da qualidade do viver e, ainda, como profissão interativa, proporciona a oportunidade de rever atitudes na relação com os pacientes $^{(4)}$.

Uma aproximação para conhecer esse processo de socialização é o objetivo deste estudo, ou seja, analisar a produção científica em enfermagem pediátrica no
Brasil, por meio do levantamento e análise de resumos publicados em periódicos, teses e dissertações nacionais no período de 2000 a outubro de 2007.

\section{REFERENCIAL TEÓRICO}

Como campo de saber específico, o enfermeiro que atua em pediatria necessita de argumentação com bases científicas a fim de consolidá-lo e validar o já existente, buscando mudanças necessárias às demandas da prática profissional. Quando os enfermeiros fundamentam sua prática na ciência, na pesquisa, no registro e divulgação de resultados clínicos, eles validam suas contribuições a todos os envolvidos, clientes, instituições e principalmente à profissão de enfermagem ${ }^{(5)}$. Nesse contexto, quando a pesquisa está presente no cotidiano de enfermeiros há maior reflexão acerca de sua prática, bem como a observação de fenômenos desvelados, facilitando a alteração de uma realidade, caracterizando a Enfermagem como disciplina do saber.

Sobre a realidade da produção científica em enfermagem pediátrica, um estudo acerca da temática Saúde da Criança nos Congressos Brasileiros de Enfermagem (CBEn) dos anos 70, mostrou-se pouco abordada. Dentre os temas relacionados à saúde da criança encontraram 10 (5,07\%) entre 197 temas oficiais e 17 (7,48\%) em 227 temas livres, com destaque para práticas em ambiente hospitalar ${ }^{(2)}$.

Também, na década de 90, estudo em 13 periódicos mostrou 114 artigos com conteúdos de pediatria $^{(6)}$. Em outra pesquisa, nas publicações de 16 periódicos de enfermagem de 1932 a 1995 foram encontrados 435 artigos de enfermagem pediátrica. Houve prevalência de artigos descritivos exploratórios produzidos por docentes e, entre os temas desses artigos, prevalecem os que se referem aos recém nascidos. Nos discursos surgiram múltiplos conceitos sobre criança, saúde, doença e assistência de enfermagem sob influência do modelo biomédico dominante na época, visualizando-os de forma fragmentada e reducionista. Ainda que insipiente, surgiram conceitos fundamentados nos modelos holísticos, ecológico, sócio-antropológico, entre outros ${ }^{(7)}$.

Em 2003, para identificar a incidência de artigos de enfermagem geral e de saúde da criança em periódicos de enfermagem, foram analisadas as publicações em quatro periódicos das regiões sul e sudeste do país, classificados pelo Qualis da Coordenação de Aperfeiçoamento de Pessoal em 
Nível Superior (CAPES) como nacional B, internacional B e internacional C. Do total de 274 artigos, 247 eram com temáticas de enfermagem geral e 27 de saúde da criança $^{(8)}$.

Sobre a relevância destas constatações, quanto à produção científica em enfermagem pediátrica, estudo realizado em 2005 em saúde da criança e do adolescente no Brasil, utilizando referenciais da Organização Mundial de Saúde, a Política Nacional de Ciência, Tecnologia e Inovação em Saúde e a Agenda Nacional de Prioridades de Pesquisa em Saúde, mostrou que é ainda alta a taxa de mortalidade infantil no país, logo são necessários mais estudos multidisciplinares e interinstitucionais, para melhor atender as necessidades dessa população, ampliando a produção do conhecimento nessa área ${ }^{(9)}$.

Outro aspecto de análise é o fato de a produção científica ser predominantemente realizada por docentes e a participação de enfermeiros assistenciais menor. Os enfermeiros assistenciais não têm ainda se representado quanti-qualitativamente na comunidade científica de Enfermagem. Sua potencialidade não tem sido devidamente explorada para anunciar a criatividade e as inovações de sua prática, fazendo com que essa comunidade não tome conhecimento e/ou não tenha imagens mais definidas desse profissional ${ }^{(10)}$.

Fator importante, também, é a pouca interação que ainda ocorre entre as instituições de ensino e os hospitais no apoio à efetivação das pesquisas, pois cuidar é elemento chave das dimensões ensinar e pesquisar problemas reais, concretos e objetivos da prática. Ambos precisam estar entrelaçados, sendo necessário o retorno dos estudos à prática assistencial, nos quais os resultantes destes, devem ser aplicadas no cotidiano ${ }^{(11)}$.

A atualidade mostra que as pesquisas em enfermagem devem permitir ao enfermeiro aliar o conhecimento técnico, ao conhecimento humanístico e científico, no entanto ainda falta valorizar o seu fazer, fundamentar as ações de cuidar em conceitos teóricofilosóficos, clínicos e técnicos e aliar o seu fazer ao saber e ao saber fazer, buscando a integração na própria ação do cuidar ${ }^{(12)}$.

Em enfermagem pediátrica as práticas associadas ao saber científico consistente por meio de projetos significativos, viáveis e de credibilidade fundamentam e qualificam o cuidado dirigido à criança. Alerta-se que o avanço profissional da construção científica impõe-se, sobretudo pela pesquisa e a Enfermagem, que tem raízes científicas, necessita, então, dar testemunho de sua atualidade na história e ciência de seu tempo. Fato efetivado por observação, aplicação de princípios, utilização de métodos e técnicas, formalidades de esquemas de trabalho, domínio de conceitos reguladores da ação que permitem analisar e criticar o modelo de ensino e prática ${ }^{(13)}$.

\section{METODOLOGIA}

Constituiu-se de pesquisa descritiva exploratória, realizada em bancos de dados disponíveis on line, utilizando os descritores: enfermagem, enfermeira, criança, pré-escolar, escolar e pediatria, aceitos pelo sistema Ciências da Saúde, adotado pela BVS Biblioteca Virtual em Saúde.

Os bancos de dados pesquisados foram as bases eletrônicas LILACS (Literatura Latino-Americana e do Caribe em Ciências da Saúde) e BDEnf (Base de dados da Enfermagem) e SCIELO (Scientific Electronic Library Online) com a intenção de resgatar os trabalhos que tratassem de enfermagem pediátrica. É provável que existam outras pesquisas e autores que não estão contemplados neste levantamento, por isso não se tem a pretensão de considerá-lo completo.

A coleta de dados compreendeu dois procedimentos: levantamento bibliográfico em bancos de dados e análise dos resumos selecionados segundo: título do periódico, tipo e ano de publicação e área temática. Tomou-se como referência o conceito de resumo como breve descrição de um trabalho científico, permitindo visão geral do conteúdo para avaliar se correspondia ao objetivo da investigação.

Foram excluídos os resumos com descritores: criança prematura, recém nascido, lactente, neonatologia, saúde materno infantil, berçário, puericultura, amamentação, assistência obstétrica e perinatal. O propósito destas exclusões foi centrar atenção às pesquisas relacionadas aos serviços de saúde em pediatria. Excluíu-se também, publicações repetidas quando possuíam, primeiramente, o mesmo título, embora sendo publicadas em fontes e anos diferentes.

Após o levantamento foi realizada leitura exploratória dos resumos, selecionando-se os artigos pertinentes ao objeto pesquisado com base nos seguintes critérios: ano de publicação entre 2000 a outubro de 2007; publicação em língua portuguesa, referindo-se a aspectos brasileiros e resumo que possibilitassem uma visão geral do conteúdo.

Para a identificação e análise de dados foram criadas planilhas em Excel contendo título do periódico; ano de publicação; tipo de publicação: artigo, tese, 
dissertação e área temática.

A classificação das áreas temáticas dos resumos foi baseada em produção científica de $1993^{(14)}$, com algumas adaptações: Assistencial: trabalhos sobre ações de cuidado de enfermagem à criança, familiares, grupo ou comunidade. Administrativa: trabalhos sobre planejamento, organização, direção e supervisão da assistência de enfermagem; avaliação dos aspectos organizacionais da assistência; estudo sobre a avaliação das unidades de serviços de saúde, de enfermagem e auditoria. Ensino e pesquisa: trabalhos relacionados ao ensino e pesquisa em enfermagem pediátrica em graduação, especialização, mestrado e doutorado que envolva aplicação de teorias e conceitos de enfermagem e outras áreas, bem como elaboração e aplicação de instrumentos, metodologias e validação de instrumentos de coleta de dados; Profissional: trabalhos sobre o objeto de trabalho da enfermagem, recursos humanos e mercado de trabalho, autonomia profissional, integração docente-assistencial, divisão social do trabalho, enfermagem e políticas de saúde, ética e legislação, produção, divulgação e utilização do conhecimento em enfermagem pediátrica; Reflexões Teóricas: trabalhos relacionados ao estudo e/ou reflexões sobre teorias e conceitos de enfermagem e outras áreas. Foi acrescida a categoria Descrição de realidade, fenômeno e experiência como subsídio para qualquer uma das outras categorias. Nela estão os depoimentos, experiências, relatos de familiares, mães, irmãos, pais, acompanhantes, pessoas da comunidade sobre algum tema relacionada à criança.

\section{RESULTADOS E DISCUSSÃO}

Foram selecionados 237 resumos e desconsiderou-se aqueles cujos títulos ou conteúdos permitiram concluir que não se tratavam ou não se relacionavam à questão específica da investigação. Desse total 179 eram artigos em periódicos, 46 dissertações de mestrado e 09 teses de doutorado.

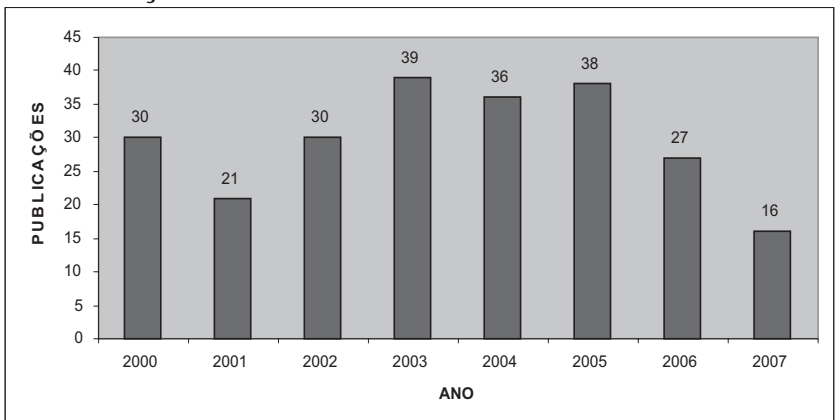

Figura 1 - Resumos publicados entre janeiro de 2000 e outubro de 2007, Curitiba, 2007
A distribuição das publicações ao longo do período estudado mostra que são produzidos em torno de 30 artigos ao ano, com elevação a partir de 2003, talvez por maior consolidação de linhas de pesquisa em cursos de Pós-Graduação, o que favorece a divulgação de temáticas em pediatria. As publicações de 2006 e 2007 tendem a ser maiores considerando que muitos artigos ainda serão editados por periódicos com volume a serem divulgados.

Um estudo documental da produção científica em enfermagem pediátrica publicada em 16 periódicos brasileiros de enfermagem de 1932 a 1995 mostrou que no período de 63 anos foram publicados 435 artigos com participação de 890 autores $^{(7)}$.

Em relação ao impacto dos estudos qualitativos de enfermagem em saúde da criança uma investigação revelou que os autores apropriam-se de referenciais teóricos das ciências biológicas, sociais, comportamentais e de enfermagem para interpretar os seus achados, contudo as citações de enfermagem em saúde da criança produzem pouco impacto na geração de novos conhecimentos quando comparadas com outras áreas ${ }^{(8)}$.

O gráfico 2 mostra a distribuição dos resumos nos periódicos nacionais indexados.

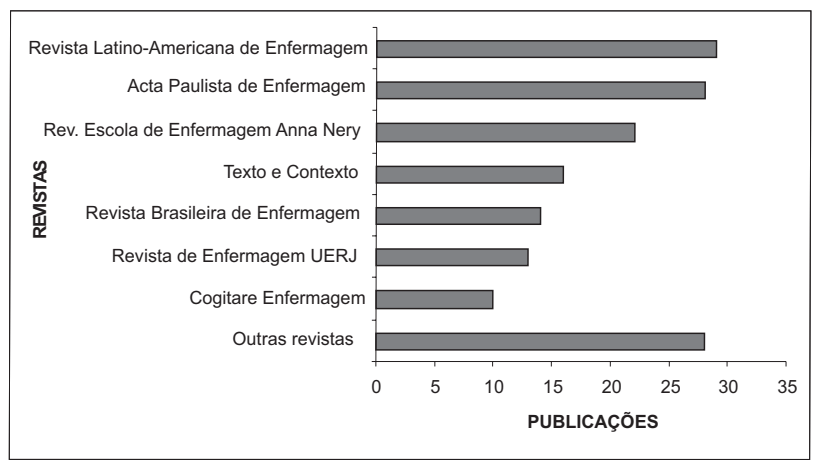

Figura 2 - Distribuição de 160 resumos publicados em revistas de enfermagem, Curitiba, 2007

A escolha dos autores pela Revista Latino Americana de Enfermagem, com o maior número de publicações, deve-se provavelmente pela rigorosa periodicidade e maior número de volumes ao ano. Em seguida a Revista Acta Paulista, deve-se provavelmente por possuir grupos consolidados de pesquisa na área de enfermagem pediátrica como: GEBrinq - Grupo de Estudos do Brinquedo que desde 1994 desenvolve estudos, eventos, assessoria e prática assistencial propriamente dita, relativa à utilização do Brinquedo/Brinquedo Terapêutico nas diferentes situações de assistência à criança e sua família; 
NECAd - Núcleo de Estudos da Criança e do Adolescente nas linhas de pesquisa: Teorias, métodos e instrumentos do cuidar em enfermagem; O cuidar de criança, adolescente e família e/ou grupos em situações específicas de agravos a saúde; Processo de ensino e aprendizagem para o cuidar; SEGTEC Segurança e Tecnologia - Grupo de pesquisas de enfermagem em segurança do paciente, cuidados intensivos e terapia intravenosa em pediatria, nas linhas de pesquisa: Enfermagem em cuidado intensivo pediátrico; Estudos de métodos, intervenções e tecnologias aplicadas ao cuidado de enfermagem para a promoção de segurança do paciente; Terapia intravenosa, intravascular e medicamentosa em pediatria; Estudos com famílias de crianças em cuidados intensivos ou especializados de enfermagem.

A essa constatação soma-se que há tendência de expansão nacional e regional na produção científica em enfermagem pediátrica na medida em que se criam cursos e programas de mestrado e doutorado, ainda com hegemonia da Região Sudeste ${ }^{(17)}$.

Dentre outras revistas somaram-se 28 publicações assim distribuídas: Revista Eletrônica de Enfermagem; Revista da Escola de Enfermagem USP; Online Brasilian Journal e Revista Gaúcha de Enfermagem. Outros 19 resumos foram publicados em outras revistas da área da saúde: Revista Brasileira de Crescimento e Desenvolvimento Humano; Mundo da Saúde; Revista Brasileira de Saúde Materno Infantil.

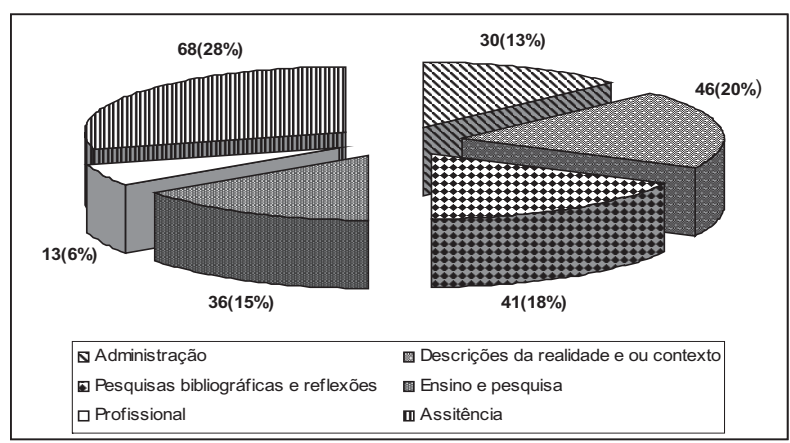

Figura 3 - Áreas temáticas dos resumos publicados, Curitiba, 2007

A área temática de maior interesse dos enfermeiros pediatras foi assistência (28\%), envolvendo primordialmente os cuidados dirigidos à criança, com extensão para questões de participação de mães no tratamento e o envolvimento de ambos com as equipes de enfermagem. Achado semelhante com crianças em serviço de oncologia sinaliza para estudos que buscam compreender a experiência das crianças, famílias e equipes hospitalares. Os autores alertam que a doença abrange faces física, social e psicológica, logo, o atendimento à criança deve ser global ${ }^{(15)}$.

Como em outra pesquisa, este estudo também relevou que as temáticas pesquisadas têm maior preocupação na investigação sobre o saber e o fazer no campo da assistência. Contudo, há necessidade de investigações em outras esferas temáticas ${ }^{(17)}$.

Uma delas, por exemplo, nesta pesquisa, foi administração com 30 estudos (13\%). Observou-se que tratam prioritariamente de dinâmicas internas de serviços, no entanto, para se conhecer e poder atuar efetivamente é preciso conhecer a situação de realidade macroestrutural, caso contrário a visão de um contexto se torna reduzida. O conhecimento do entorno possibilita maior lastro para tomada de decisões e, sobretudo para interpretações analíticas concomitantes com a teoria.

A pesquisa mostrou 46 resultados de descrições de realidade e, ou situações do cotidiano (20\%), caracterizados nesta pesquisa como estudos descritivos, que são significativos pela sua configuração, mas têm pouco impacto na prática, por descreverem uma realidade sem a efetiva transformação dela. Por outro lado, com configuração semelhante, houve 41 (18\%) estudos teóricos que sempre se mostram relevantes por permitir tomar conhecimento do estado da arte de alguma temática.

Com menores percentuais aparecem $36(15 \%)$ resumos tratando de ensino e pesquisa e 13 (6\%) em assuntos profissionais. Essa última temática é pouco explorada em outras especialidades em enfermagem, ficando aquém dos requerimentos das associações de classe de enfermagem. Quanto ao ensino e pesquisa pode-se, talvez, inferir que os docentes têm maior preferência pelos temas, mas os desenvolvem no conjunto das disciplinas dos currículos, quando nos estudos sobre formação profissional, projetos pedagógicos e avaliações institucionais.

\section{CONCLUSÃO}

O estudo mostrou que de 237 resumos entre 2000 a outubro de 2007, há uma média de pouco mais de 30 artigos por ano, o que permite concluir que há espaço para ampliação da visibilidade da enfermagem pediátrica no cenário da produção científica nacional.

A classificação por área temática mostrou que a administração em enfermagem foi a que recebeu o menor número de publicações. Importante destacar que essa área possibilita e favorece a atuação profissional, permitindo e facilitando que outras áreas possam organizar, executar, avaliar e divulgar

Cogitare Enferm 2009 Jan/Mar; 14(1):144-9 
metodologias de trabalho. Outro aspecto foi um significativo número de trabalhos envolvendo a tríade: criança, mãe e equipe de enfermagem, nos caminhos de maior compreensão de experiências desses envolvidos na assistência.

Conclui-se que a produção científica transforma a prática e certamente possibilita maior credibilidade para tomada de decisões. É pelo conhecimento sistematizado, bem como ancorado nas experiências profissionais que as enfermeiras pediátricas podem e devem revelar mais seus atributos de competência, de empatia e de responsabilidade nos serviços em que atuam.

\section{REFERÊNCIAS}

1. Almeida AM, Oliveira ERA, Garcia TR. Pesquisa em enfermagem e o positivismo. Esc Enferm USP. 1996;30(1):25-32.

2. Costa RO, Oliveira ICS. Produção científica dos Congressos Brasileiros de Enfermagem nos anos 70: contribuições para enfermagem pediátrica. Rev Eletron Enferm. 2006;8(1):83-90.

3. Neves EP, Souza IEO. Pesquisa em enfermagem: buscando resgatar a posição do sujeito que a desenvolve. Texto Contexto Enferm. 2003;12(3):387-93.

4. Castanha ML, Lacerda MR, Zagonel IPS. Hospital: lugar para o enfermeiro cuidar do imaginário?. Acta Paul. Enferm [periódico na Internet]. 2005 Mar [citado 2008 Maio 16] 18(1):94-99. Disponível: www.scielo.br.

5. Wong DL. Enfermagem Pediátrica. $7^{\mathrm{a}}$ ed. Rio de Janeiro: Elsevier; 2006.

6. Silva FD, Cabral, IE. O cuidado de enfermagem à criança no pós-terapia intensiva pediátrica: reflexos na produção científica da década de 90. Esc Anna Nery Rev Enferm. 2001;5(1):93-103.

7. Kakehashi S. Enfermagem pediátrica brasileira: produção científica de 1932 a 1995 [tese]. São Paulo (SP): Escola de Enfermagem Universidade de São Paulo; 1998.

8. Cabral IE. O impacto dos estudos qualitativos de enfermagem em saúde da criança. Rev Enferm UERJ. 2006;14(1):61-6.

9. Rodrigues BMRD, Christoffel MM, Cunha JM, Pacheco STA, Reis CSC. Tendências da pesquisa na saúde da criança e os desafios para a enfermagem brasileira. Rev Enferm UERJ. 2005;13(1):112-6.
10. Dyniewicz AM. Metodologia da Pesquisa para enfermeiras: práticas educativas em hospital universitário [tese]. São Paulo (SP): Universidade Federal de São Paulo. Escola Paulista de Medicina. Programa de Pós-graduação em Enfermagem; 2003.

11. Daher DV, Espírito Santo FH, Escudeiro CL. Cuidar e pesquisar: práticas complementares ou excludentes? Rev Latino-Am Enferm. 2002;10(2):145-50.

12. Neves EP, Zagonel IPS. Pesquisa-cuidado: uma abordagem metodológica que integra pesquisa, teoria e prática em enfermagem. Cogitare Enferm. 2006 Jan/ Abr;11(1):73-9

13. Carvalho V. Tendências da pesquisa na enfermagem: aspectos epistemológicos e outras implicações. Dezembro, 2000. (mimeo)

14. Assis MMA, Nascimento MAA, Jorge MSB, Matos MS, Braga VAB, Moreira ASP, Garcia TR. Produção científica de enfermagem na Região Nordeste: 1988-1992. Rev Latino-Am Enferm. 1993;1(n. esp):85-102.

15. Moreira GMS, Valle ERM. Estudos bibliográficos sobre publicações brasileiras relacionadas a aspectos psicossociais do câncer infantil, no período de 1980 à 1997. Rev Bras Cancerol. 1999;45(2):27-35.

16. Christoffel MM, Rodrigues BMRD. As abordagens metodológicas na pesquisa em enfermagem na área de saúde da criança e adolescente: análise da produção científica no período de 1995 a 1999. Esc Anna Nery Rev Enferm. 2002;6(supl.1):15-24.

17. Tyrrell MAR, Cabral IE. A produção científica de enfermagem em saúde da mulher e da criança: programa brasileiro. Rev Enferm UERJ. 2005;13(1):103-11. 